\title{
Modeling of Circumstellar Dust by the DUSTY Code
}

\author{
Tomislav Jurkić and Dubravka Kotnik-Karuza \\ Department of Physics, University of Rijeka, Omladinska 14, HR-51000 Rijeka, Croatia \\ email: tjurkic@phy.uniri.hr
}

\begin{abstract}
We present a circumstellar dust model around the symbiotic Mira RR Tel obtained by modeling the near-infrared JHKL magnitudes and ISO spectra. In order to follow the evolution of infrared colours in time, the published JHKL magnitudes were corrected by removing the Mira pulsations. The RR Tel light curves show three obscuration events in the near-IR. Using the simultaneously available JHKL magnitudes and ISO spectra in three different epochs, we obtained SEDs in the near- and mid-IR spectral region (1-20 $\mu \mathrm{m})$ in epochs with and without obscuration.

The DUSTY numerical code was used to solve the radiative transfer and to determine the circumstellar dust properties of the inner dust regions around the Mira, assuming a spherical dust temperature distribution in its close neighbourhood. The physical properties of the dust, mass loss and optical depth during intervals with and without obscuration have been obtained. Both JHKL and ISO observations during the obscuration period can be reproduced with a spherical dust envelope, while ISO spectra outside obscuration show a different behaviour. The dynamical behaviour of the circumstellar dust was obtained by modeling the JHKL magnitudes observed during the span of more than 30 years.

The DUSTY code was also successfully applied in the modeling of circumstellar dust envelopes of young stellar objects, such as Herbig Ae/Be stars.
\end{abstract}

Keywords. stars: binaries: symbiotic, stars: circumstellar matter, stars: AGB and post-AGB, infrared: stars, methods: numerical, radiative transfer

\section{Introduction}

Symbiotic stars are interactive binary systems consisting of a cool red giant or a Mira star, and a hot compact star, such as white dwarf or neutron star. Around $20 \%$ of symbiotic stars, called D-type symbiotics, consist of a Mira component with a circumstellar dust shell at the temperature of around $1000 \mathrm{~K}$ (Mikolajewska et al. 1988, Kenyon 1986). Obscuration events in the near-IR seen in most of the D-type symbiotics are probably caused by a change in optical depth of the dust shell (Kotnik-Karuza et al. 2006, Whitelock 2003).

RR Tel is a symbiotic nova that underwent its last outburst in 1944 (Whitelock 2003). A complete model of the circumstellar dust environment around RR Tel capable of explaining all near and mid-IR spectroscopic and photometric features is still to be found. Up to now, most attempts to explain the circumstellar dust environment have included only simple thick dust shell models acting as a blackbody, without determination of any physical dust properties (Kotnik-Karuza et al. 2006, Whitelock 1987). The recent colliding wind model of Angeloni et al. (2010) has proposed the existence of two blackbody dust shells at $400 \mathrm{~K}$ and $1000 \mathrm{~K}$, with maximum emission of the $400 \mathrm{~K}$ dust shell at around 10 microns, typical for silicate emission. Generally, only few authors used radiative transfer in other single objects to model the dust properties.

Young stellar objects such as T Tau or Herbig Ae/Be stars show the presence of the dust in the form of strong near and mid-IR emission (Chiang \& Goldreich 2001). Some 
of these stars, especially Herbig Ae/Be stars, have significant near-IR excess that cannot be explained with simple flared disk models, and require introduction of complex disk geometry (Dullemond et al. 2001).

\section{Observational data and DUSTY code}

Multiwavelength and long-term infrared observations are needed to completely determine the dynamical model of symbiotic Miras. In order to fulfill this demand, near-IR JHKL photometric magnitudes were collected and spectral energy distributions (SED) from $1.2 \mu \mathrm{m}$ to $20 \mu \mathrm{m}$ reconstructed. We have used all available published JHKL magnitudes from the South African Astronomical Observatory (Gromadzki et al. 2009), corrected for Mira pulsations and interstellar reddening using our own developed algorithm. The JHKL light curves show three distinct obscuration intervals (Kotnik-Karuza et al. 2006). Spectral energy distributions during and outside obscuration epochs were reconstructed using ISO short wavelength infrared spectra together with the near-IR JHKL observations at three epochs when both JHKL magnitudes and ISO spectra were simultaneously available.

The numerical code DUSTY (Ivezic, Nenkova \& Elitzur 1999) was used to model the dust properties of the circumstellar dust shell around the cool Mira component. DUSTY solves the radiative transfer through the circumstellar dust, assuming a spherical temperature distribution and using scaling invariance and self-similarity theory to constrain the number of free input parameters (Ivezic \& Elitzur 1997). Scaling invariance implies only the scaled distance $y$ with respect to the inner dust shell radius (sublimation radius), $r_{i n}$, as a relevant parameter:

$$
y=\frac{r}{r_{i n}}
$$

Only the shape of the dust density distribution and the spectral shape of the input heating radiation is needed; absolute stellar luminosity does not enter the equations. Starting from the input parameters, the code models the output radiation reprocessed by dust and gives physical and geometrical properties of the dust shell.

DUSTY can handle various analytical forms for the dust density distribution, including full dynamical calculation for radiatively driven winds. Various dust chemistry and optical properties are supported (silicates, carbon, olivine, forsterite, etc.) or can be entered in the user-supplied files. The dust grain size distribution, the minimum and maximum grain sizes, the dust sublimation temperature, the shell size, and the optical depth can be varied in order to produce an acceptable model. The variation in power index of the dust density distribution gives an insight into the dust geometry (spherical shell, flattened halo, disk-like structure).

The circumstellar model with a Mira in the centre of the dust shell was used, while input stellar radiation was approximated with a Mira blackbody at a temperature between $2200 \mathrm{~K}$ and $2800 \mathrm{~K}$, in agreement with its spectral class. As Miras are well known to have strong stellar winds which drive the expansion of their envelopes, an analytical approximation for the dust density distribution, $\eta$, enhanced by radiatively driven winds (Ivezic et al. 1999) was used:

$$
\eta \propto \frac{1}{y^{2}} \sqrt{\frac{y}{y-1+\left(v_{i} / v_{e}\right)^{2}}}
$$

where $v_{i}$ and $v_{e}$ are initial and terminal wind velocities respectively. A typical MRN dust grain size distribution (Mathis, Rumpl, \& Nordsieck 1977): $n(a) \propto a^{-q}\left(a_{\min } \leqslant a \leqslant a_{\max }\right)$ was applied in the model, with the power index $\mathrm{q}=3.5$, the minimum grain size $a_{\text {min }}$ 
Table 1. Dust parameters of RR Tel derived from DUSTY modeling $\left(T_{\text {mira }}\right.$ - mira temperature; $T_{d u s t}$ - dust sublimation temperature; $a_{\max }$ - maximum grain size; $\tau_{V}$ - visual optical depth; $A_{K}$ - extinction at $\mathrm{K}$ band; $\dot{M}$ - mass-loss rate; $v_{e}$ - terminal wind velocity)

\begin{tabular}{llll}
\hline Parameters & \multicolumn{1}{c}{$\begin{array}{c}\text { Maximum } \\
\text { Obscuration }\end{array}$} & \multicolumn{2}{c}{$\begin{array}{c}\text { Minimum } \\
\text { Obscuration } \\
\text { thin shell }\end{array}$} \\
& & \multicolumn{2}{c}{2200} \\
\hline$T_{\text {mira }}[\mathrm{K}]$ & $2500 \pm 300$ & \multicolumn{2}{c}{ thick dust } \\
$T_{\text {dust }}[\mathrm{K}]$ & $1200 \pm 100$ & $1000-1200$ & 1200 \\
$a_{\text {max }}[\mu \mathrm{m}]$ & $4.0 \pm 1.0$ & $0.5-1.0$ & $1.5 \pm 0.5$ \\
$\tau_{V}$ & $5.2-5.8$ & 0.4 & \\
$A_{K}$ & $0.58-0.64$ & 0.04 & \\
$\dot{M}\left[10^{-6} M_{\odot} / y r\right]$ & $11 \pm 1$ & 0.4 & \\
$v_{e}[\mathrm{~km} / \mathrm{s}]$ & $26 \pm 2$ & 28 & \\
\hline
\end{tabular}

fixed at $0.005 \mu \mathrm{m}$, and the maximum grain size $a_{\max }$ determined by modeling. The dust composition typical for Miras containing 100\% warm silicates has been assumed (Ossenkopf, Henning \& Mathis 1992). The outer dust shell radius was fixed at $20 r_{i n}$, while the inner dust shell radius $r_{i n}$ was obtained by fitting, together with the dust sublimation temperature $T_{\text {dust }}$.

\section{Results}

Reconstructed SEDs and two-colour near-IR diagrams during obscuration epochs are very well reproduced by a single circumstellar dust shell model of consistent sublimation temperature at $1200 \mathrm{~K}$ and a grain size of $4.0 \mu \mathrm{m}$ (Table 1). On the contrary, reconstructed SEDs in an epoch without obscuration cannot be explained by a simple single shell model as the Mira blackbody is itself almost unattenuated, while the dust emission is still strongly present. Such behaviour suggests an optically thin dust shell around the Mira component and an optically thick dust of undetermined geometry outside the line of sight, probably in a kind of toroidal configuration or something similar. Modeling of such a two-shell model yielded a very thin dust shell with optical depth around 0.4 and thick dust outside the line of sight, both at sublimation temperature around $1200 \mathrm{~K}$ and grain size of about $1.5 \mu \mathrm{m}$.

Our model shows no difference in dust sublimation temperatures between epochs with and without obscuration inside the error margin. Obscuration events can be explained by an increase in optical depth, likely to be caused by higher mass loss leading to a larger amount of dust condensed around the Mira and to the formation of a thicker dust shell. The only way to model the reconstructed SEDs and the near-IR colours requires a significant change in the maximum dust grain size from around $1.5 \mu \mathrm{m}$ to $4.0 \mu \mathrm{m}$ in transition from epochs with obscuration to epochs without obscuration. This can be explained by grain growth in the dust probably originating from the Mira itself.

In young stellar objects such as Herbig Ae/Be stars, the near-IR excess requires a larger near-IR emitting area, which can be fulfilled by use of the spherical dust shell instead of the complex disk geometry. Simultaneous modeling of SEDs and interferometric observations of different Herbig Ae/Be stars ranging in luminosities from $25 L_{\text {Sun }}$ to 90 $000 L_{S u n}$, have shown consistently that an optically thin dusty halo around the inner disk regions can very well explain the near-IR excess, with no need for complex disk geometry (Vinkovic et al. 2006). 


\section{References}

Angeloni, R., Contini, M., Ciroi, S., \& Rafanelli, P. 2010, MNRAS, 402, 2075

Chiang, E. I. \& Goldreich, P. 2001, ApJ, 490, 368

Dullemond, C. P., Dominik, C., \& Natta, A. 2001, ApJ, 560, 957

Gromadzki, M., Mikolajewska, J., Whitelock, P., \& Marang, F. 2009, AcA,

Ivezic, Z. \& Elitzur, M. 1997, MNRAS, 287, 799

Ivezic, Z., Nenkova, M., \& Elitzur, M. 1999, User Manual for DUSTY, University of Kentucky Internal Report, accessible at http://www.pa.uky.edu/ moshe/dusty

Kenyon, S. J. 1986 The symbiotic stars (Cambridge \& New York: Cambridge Univ. Press), p.295 Kotnik-Karuza, D., Friedjung, M., Whitelock, P. A., Marang, F., Exter, K., Keenan, F. P., \& Pollacco, D. L. 2006, A\& $A$ A, 452, 503

Mathis, J. S., Rumpl, W., \& Nordsieck, K. H. 1977, ApJ, 217, 425

Mikolajewska, J., Friedjung, M., Kenyon, S. J., \& Viotti, R. 1988, in: J. Mikolajewska, M. Friedjung, S. J. Kenyon \& R. Viotti (eds.) The symbiotic phenomenom, Proceedings of IAU Colloq. 103 (Dordrecht: Kluwer), vol. 145, p. 381

Monnier, J. D., Berger, J.-P., Millan-Gabet, R., et al., 2006, ApJ, 647, 444

Ossenkopf, V., Henning, Th., \& Mathis, J. S. 1992, A\&A, 261, 567

Vinkovic, D., Ivezic, Z., Jurkic, T., \& Elitzur, M. 2006, ApJ, 636, 348

Vinkovic, D. \& Jurkic, T. 2007, ApJ, 658, 462

Whitelock, P. A. 1987, PASP, 99, 573

Whitelock, P. A. 2003, in: R. L. M. Corradi, J. Mikolajewska \& T. J. Mahoney (eds.) Symbiotic stars probing stellar evolution, ASP-CS, 303, 41

\section{Discussion}

K. Allers: Your point that the puffed-up disk is not necessary if you have a dust shell does not always apply. T Tauri stars show no obscuration but do show the near-IR bump.

T. JuRkic: Yes, that is true. On the other hand, the dusty halo in the innermost parts of the CS environment is usually optically thin $\left(\tau_{V} \sim 0.5\right)$ and can reproduce T Tau spectra very well. The presence of a dusty halo does not exclude the existence of a disk which can be seen at larger wavelengths. Near-IR interferometry of T Tau stars speaks in favor of dusty halos in the regions up to $10 \mathrm{AU}$ (Vinkovic et al. 2006).

O. De Marco: Sub-mm observations can discriminate between shell and disk/torus dusty CS geometries. Have such observations been carried out for RR Tel or other symbiotics?

T. JURKIC: Such observations have been used to detect disks embedded in dusty halos around YSOs such as Herbig Ae/Be stars. Unfortunately, there was no sub-mm imaging of RR Tel. Sub-mm spectra are affected not only by the dust emission but also by the free-free emission, which further complicates analysis.

P. STEE: We have also tried to fit the SED of HAeBe stars using a disk model and it is not difficult to fit the SED, especially if you have two different dusty disks. Regarding the interferometric data, you are mainly constraining the flux ratio between the central star and the disk rather than the global geometry since you need a better plane coverage.

T. JuRKIC: It is true that better plane coverage will constrain dust geometry better. Monnier et al. (2006) detected very small deviations from centrally symmetric images using near-IR interferometry, which is not consistent with inclined disk models. Modeling of the near-IR visibilities of 40 YSO (Vinkovic et al. 2007) showed that T Tau and low luminosity Herbig Ae/Be stars are best explained by an inner dusty halo. 